\title{
Actualidad en el agro colombiano y aprovechamiento de los recursos visto desde la robótica
}

Yeyson Alejandro Becerra Mora*, Sebastián Soto Gaona**

\section{Resumen}

Diversos informes sobre el crecimiento de la población y la demanda de alimentos a nivel mundial señalan que es indispensable realizar una gestión más eficiente del suelo, para tener un mayor rendimiento por metro cuadrado cultivado. Muchos países tienen un gran potencial para explotación agrícola a gran escala, que luego serán llamadas las despensas del mundo, entre ellas Colombia. A partir del análisis de la panorámica nacional del agro colombiano, se proyectan diversas soluciones desde el punto de vista de la robótica y su relación directa con agricultura de precisión. La robótica desempeña un papel importante como maquinaria especializada en la realización de tareas complejas, al asistir al hombre y ayudarlo a obtener un mayor rendimiento de los recursos. Las proyecciones globales para la robótica en la agricultura se muestran rentables y prometedoras, gracias a los diversos avances de esta tecnología.

Palabras clave: agricultura de precisión, autónomo, robótica

\begin{abstract}
Several reports on population growth and demand for food worldwide point out that more efficient soil management is indispensable for higher yields per square meter cultivated. Many countries have great potential for large scale agricultural exploitation, that will be called the pantries of the world, and Colombia is one of them. From the analysis of the national panorama of Colombian agriculture, various solutions are projected from the point of view of robotics and its direct relationship with precision agriculture. Robotics plays an important role as specialized machinery in carrying out complex tasks, by assisting man and helping him to obtain a greater yield of resources. The global projections for robotics in agriculture are both profitable and promising, thanks to the diverse nature of this technology.
\end{abstract}

Keywords: autonomous, precision farming, robotics

* Ingeniería Mecatrónica, Fundación Universitaria Agraria de Colombia - Uniagraria Contacto: becerrayeyson@gmail.com

** Ingeniería Mecatrónica, Fundación Universitaria Agraria de Colombia - Uniagraria Contacto: soto.sebastian@uniagraria. edu.co 


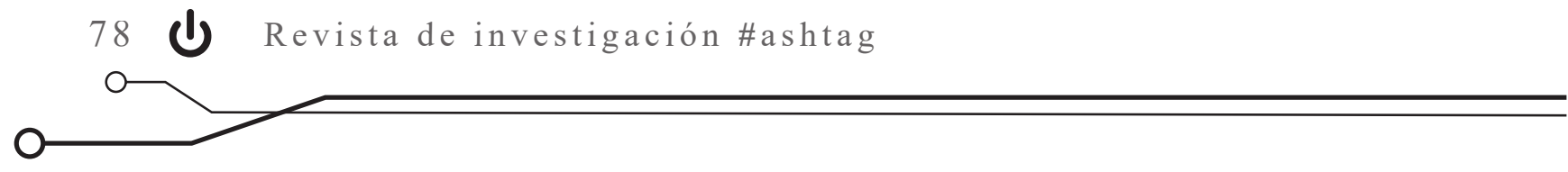

\section{Introducción}

Una vez finalizó el conflicto armado con las Fuerzas Armadas Revolucionarias de Colombia (FARC), la agroindustria colombiana comenzó a perfilarse como uno de los sectores económicos más prometedores del país. Esto se debió a que las zonas que fueron ocupadas por el conflicto armado comenzaron a ser reutilizadas para la producción de alimentos, que no fueron utilizadas por décadas debido a este flagelo.

Sin embargo, se debe realizar un trabajo social fuerte que permita la restitución de tierras y que más campesinos vuelvan a sus actividades. Según un informe de las Naciones Unidas para los Refugiados (ACNUR), Colombia en el 2016 ocupada el primer lugar con más de 7,4 millones de desplazados internos.

\section{Actualidad en el agro colombiano}

En el 2016, se evidenció que 26 millones de hectáreas tienen capacidad de producción agrícola, pecuaria y forestal; de las cuales solo se aprovechaba el 24,2 \%. De los suelos netamente agrícolas, que son 11,3 millones de hectáreas, solo se aprovechaba el 34,2 \% (Morales, 2016). Igualmente, se pudo evidenciar que, debido a esta falta de aprovechamiento de terreno apto para la agricultura, se estaba importando alrededor del $28,5 \%$ de alimentos consumidos en los hogares. Una vez finalizó el conflicto armado en este mismo año, se previó el gran potencial de Colombia en la agroindustria, siendo este uno de los sectores con mayor futuro y que más le aportaría al PIB del país, más aún cuando las
Otro problema identificado en el sector rural ha sido el envejecimiento de la población en los últimos años. Cada vez con mayor frecuencia, los jóvenes de entre los 20 y 30 años migran a las urbes con la esperanza de conseguir mejores oportunidades. Además, el promedio de integrantes por hogar en el sector rural ha disminuido de 4 a 3,32 personas (Unimedios, 30 de diciembre de 2015). Inclusive, padres agricultores prefieren que sus hijos reciban educación universitaria y migren a las ciudades en lugar de permanecer en el campo.

Debido a la gran cantidad de intermediarios, desde el productor hasta el consumidor final, el producto puede llegar a costar entre 2 y 5 veces más. Por lo tanto, el beneficio del agricultor se ve afectado considerablemente. Dado lo anterior, el campesino colombiano ha venido desistiendo en dejar su legado a futuras generaciones.

proyecciones de producción y reservas en el sector petrolero se encuentran a la baja.

El peso que tuvieron los productos agrícolas sobre las exportaciones realizadas en el 2017 fue de tan solo el $8 \%$, siendo el café y el banano los productos más representativos (BLOOMBERG, 2017); mientras que en países como Chile y Argentina, la exportación de productos agrícolas pesó el 14 \% y 50 \% respectivamente, del total de las exportaciones realizadas en ese mismo año.

Las proyecciones que se tienen para la población mundial en el 2050 se sitúan en torno a las 9.000 millones de personas $y$, según lo manifiesta el 
Fondo Internacional para el Desarrollo Agrícola (IFDA), la producción de alimentos en los países en vías de desarrollo tendrá que duplicarse. Acorde a estas proyecciones, la Organización de las Naciones Unidas para la Alimentación y la Agricultura, FAO, afirma que Colombia puede llegar a ser una potencia en el sector agroindustrial si se hacen los debidos ajustes en este momento. Según datos de la FAO, Colombia es el cuarto país de Latinoamérica con tierras disponibles para la producción de alimentos, y el tercero con mayores recursos de agua, diversidad climática y tasas de precipitación anual. Además, los informes internacionales señalan que los alimentos han de ser suministrados por tierras más productivas, las cuales representaran el 80 \% del parque agrícola disponible y solo el $20 \%$ será equivalente a nuevas tierras. Por lo tanto, es indispensable transformar el primer sector con mejoramiento genético, desarrollo de tecnologías de punta, agricultura de precisión, economía sustentable, automatización de procesos, integración de mercados e innovación, entre otros (Fondo para el Financiamiento del Sector Agropecuario, Finagro, 2018).

Si bien se está ante una oportunidad inmensa de incrementar los niveles de producción agrícola, aún falta un largo camino por recorrer. A los problemas que enfrenta el campesino colombiano, como los mencionados previamente, se le suman los siguientes: informalidad en la tenencia de la tierra, altos costos logísticos y bajos niveles de inversión en tecnología para el sector agroindustrial. Con el acuerdo de paz firmado en el 2016, se avanzó considerablemente en la solución de algunos de estos problemas, al hacer volver al campesino a las áreas rurales por medio de la restitución de tierras, con incentivos para que permanezca en ellas por medio de programas sociales.
Otro avance hecho en los últimos años fueron los proyectos de infraestructura lanzados por el Gobierno Nacional, que establecieron nuevas vías para el mejoramiento de la competitividad del país y la disminución de costos logísticos. La inversión en tecnología en la agroindustria es aún incipiente, debido, principalmente, a los problemas de conflicto armado vividos en zonas rurales. Sin embargo, el panorama es distinto actualmente, por lo cual se espera que la inversión y la producción en la agroindustria comiencen a crecer considerablemente en los próximos años.

Asimismo, la inversión en ciencia y tecnología en el sector agropecuario es de vital relevancia para que este crezca a tasas significativas. Según lo comenta Rafael Mejía, presidente de la Sociedad de Agricultores de Colombia, SAC, se deben generar políticas de largo plazo en asistencia técnica integral que contemplen la investigación e innovación para el sector (Mejía, 2016). Un informe publicado por el Departamento Nacional de Planeación, DNP (2015) informa que las fuentes de inversión pública para actividades de I+D en el sector agropecuario dependen del Ministerio de Agricultura y Desarrollo Rural (MADR), de los Fondos Parafiscales, de Colciencias y del Fondo de Ciencia, Tecnología e Innovación (FCTI) del Sistema General de Regalías (SGR). Los recursos asignados para I+D en el sector agropecuario se vieron incrementados 159 \% en el 2011 con respecto al año anterior, gracias a los fondos que entraron por el SGR, el cual destinó 10 \% para actividades de ciencia y tecnología. Otros recursos administrados por las entidades nombradas previamente provenían del Presupuesto General de la Nación (PGN), recursos internacionales y parafiscales.

Durante la crisis en los precios del petróleo, los recursos asignados al SGR se vieron reducidos considerablemente, por lo que las actividades 


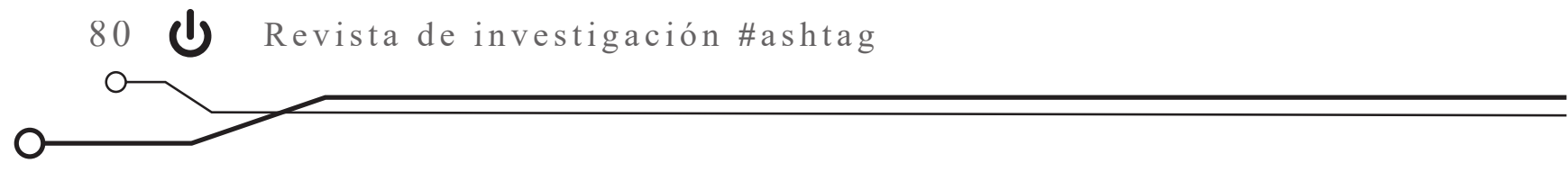

de ciencia y tecnología que dependían de fondos públicos se vieron afectadas. Sin embargo, en la actualidad el precio del petróleo se ha recuperado en más del 100 \% desde su nivel más bajo en 2016, lo que lleva a mayores recursos para I+D.

\section{La robótica como herramienta en el sector agropecuario}

La agricultura, como una de las actividades más importantes en la historia de la humanidad, está enfrentándose a nuevos desafíos. Por un lado, debe suplir la demanda alimenticia a una población de 9.000 millones de personas para el 2050; por el otro, incrementar en un $25 \%$ la eficiencia en la producción agrícola, debido a las limitaciones en tierra, agua y recurso humano. Atendiendo estas proyecciones, países como Estados Unidos y China están empezando a comprar tierras para producción agrícola en otros países. Los agricultores alrededor del mundo están empezando a utilizar métodos de agricultura de precisión: Japón, por ejemplo, realiza el $90 \%$ de sus fumigaciones con pequeños helicópteros no tripulados, y diversos países alrededor del mundo están previendo la disminución de mano de obra en el sector agropecuario para los próximos años (Keane, 2013).

En las últimas seis décadas, los robots industriales han incrementado los niveles de eficiencia y producción en el sector industrial y han reducido costos en este tipo de productos. En el sector agropecuario se está viendo una tendencia semejante. Durante los últimos 20 años se ha visto en la agricultura el uso de Global Position System (GPS), así como cosechadoras y tractores

\section{Agricultura de precisión y robótica}

El sector agrario se está beneficiado por los desarrollos tecnológicos de los UGV y UAV. La utilización de vehículos autónomos para asistir al auto guiados. Ya en los últimos años, los agricultores han empezado a experimentar con sistemas autónomos que permitan optimizar procesos de cosecha, corte, eliminación de maleza, poda, fumigación, entre otros. Sistemas como los Unmanned Aerial Vehicle (UAv) están siendo usados para mapear, observar, sensar y rociar; mientras que los Unmanned Ground Vehicle (uGV) están proporcionando movimientos más precisos en los cultivos (Gage, 1995).

Debido a los avances vistos en la robótica industrial y la ausencia de humanos en las fábricas, se teme actualmente que la robótica haga lo mismo en el sector agropecuario. Sin embargo, a pesar de que la robótica se presenta como una solución a las labores agrícolas, la FAO afirma que este es aún un mercado inmaduro. Además, es cierto que mucho del sector agropecuario se ha automatizado, pero no en su totalidad, por lo que aún se necesita de humanos en diversas tareas agrícolas. La cuarta revolución industrial cataloga a la robótica como una tecnología disruptiva, que inicialmente tomará millones de empleos en el mundo con el pasar de los años, pero que, a la larga, generará muchos más, lo que da lugar a industrias y ocupaciones que aún no existen (FAO, 2017).

agricultor permite una mayor productividad en un porcentaje menor de tierra (Fernández, 2012). Este concepto va ligado a la agricultura de 
precisión, la cual se caracteriza por gestionar de forma eficiente el manejo de la variabilidad del cultivo y del terreno en el tiempo, empleando herramientas de control y análisis de datos.

El suministro de insumos se realiza tomando en cuenta la variación en los componentes del suelo (acidez, humedad, características topográficas, relieve del terreno), con la finalidad de mejorar las condiciones de desarrollo vegetal en temporadas de siembra. La diferencia entre la agricultura de precisión y la agricultura convencional radica en que la convencional considera las condiciones de siembra como homogéneas en el terreno, mientras que la de precisión toma en cuenta la variabilidad del espacio del suelo en el tiempo, para maximizar el rendimiento y reducir los costos de inversión y consecuencias ambientales (Oficina de información científica y tecnológica para el congreso de la Unión, 2018).

La agricultura de precisión se desarrolla en tres etapas:

Figura 1. Etapas de la agricultura de precisión

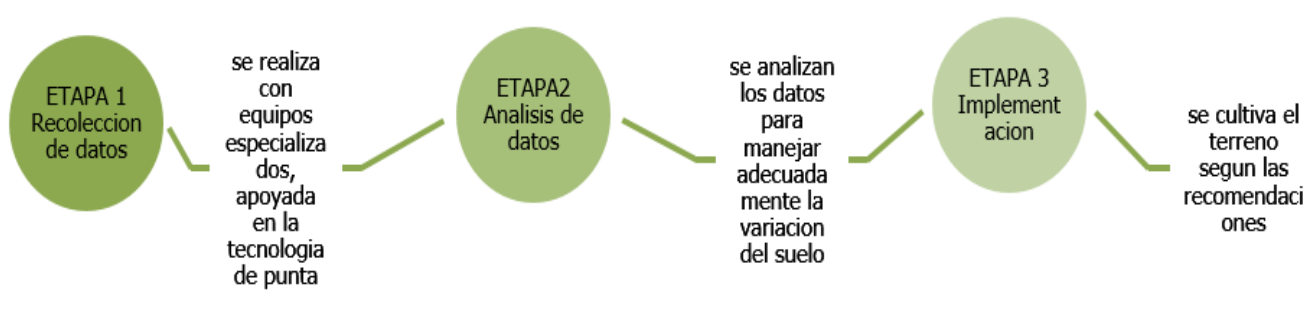

Fuente: elaboración propia.

En la agricultura de precisión existen una serie de tecnologías asociadas que potencializan sus cualidades y permiten el uso eficiente de los recursos, dichas tecnologías son:

Figura 2. Tecnologías asociadas a la agricultura de precisión

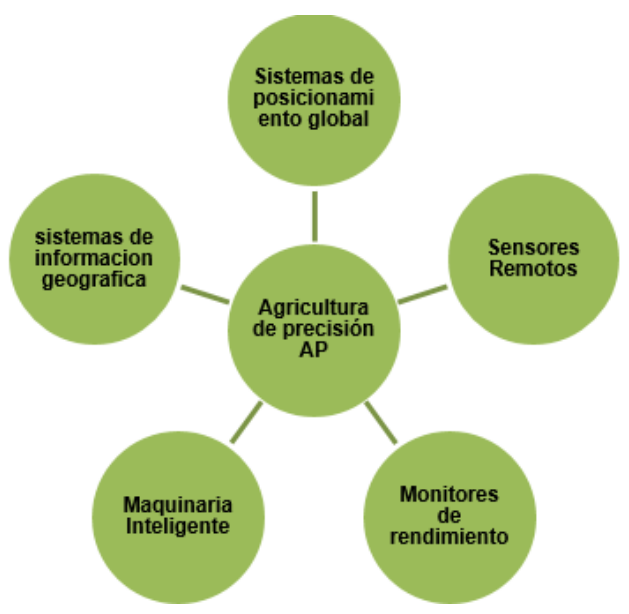

Fuente: elaboración propia. 


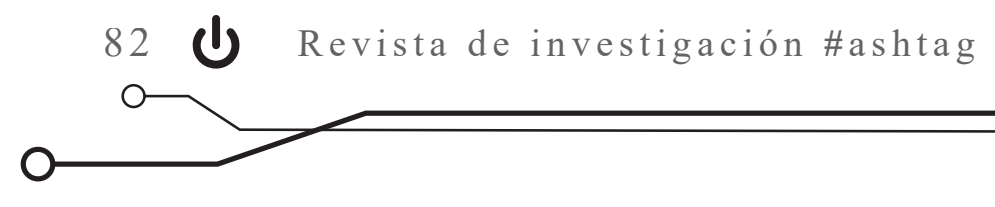

Los UAV han hecho en los últimos años que el sector agropecuario sea más eficiente y productivo. Algunos beneficios que ofrecen estos sistemas son los siguientes: obtención de información topográfica en la delimitación de áreas, actualización cartográfica, cuantificación de áreas infestadas por malezas, cuantificación de áreas de resiembra y digitalización de líneas de surcado. También se obtienen modelos digitales de elevación para estimar curvas a nivel, construcción de mapas de pendientes, modelos de superficie y modelos para estimar la altura del cultivo, entre otros.

En el sector agropecuario, los UAV pueden ser teleoperados o autónomos; su hardware puede incluir cámaras visuales, infrarrojas y/o multiespectrales o térmicas. Las cámaras visuales permiten tomar fotos georreferenciadas para identificar área efectiva, caminos, agua y realizar la logística de un amplio terreno. La información puede ser almacenada para ser consultada posteriormente $\mathrm{y}$, mediante algoritmos de visión por computador, se puede calcular la densidad de siembra y cantidad de árboles y plantas de forma automática. Las cámaras multiespectrales obtienen los colores que refleja la planta a través de una firma espectral, la cual está compuesta por características únicas e inequívocas de la planta. Con esto se identifica si la planta está saludable, con estrés hídrico o si tiene alguna maleza. Además, con las cámaras multiespectrales, se pueden generar mapas de diagnóstico, como pueden ser de condiciones de cultivo, de fertilizantes, de contenido de nitrógeno, de estrés hídrico y de plagas (Granados, 2013).

Labores como fumigar, segar, recoger, plantar, labrar; pueden ser realizadas actualmente por los UGv. La fumigación de un cultivo puede ser puntual, e incluso si el robot detecta malas hierbas puede extraerlas de forma mecánica, lo que evita el uso de peligrosos herbicidas. La siega realizada por un robot se ha visto empleada en campos de maíz en Estados Unidos, lo que ha reducido los tiempos destinados a esta labor. La recolección de frutos es una tarea un poco más complicada, ya que el robot debe emplear algoritmos de visión y procesamiento de imágenes que le permita identificar características del fruto para decidir si es momento de recolectar o no. Además se deben implementar grippers en su brazo robótico, que permitan la correcta manipulación del fruto, evitando el maltrato. En las labores de plantación y labrado, se valora la precisión de estas tareas realizadas por el robot pues así se optimizan los espacios en el cultivo.

Los UGV se caracterizan por ser robots móviles, con sistemas de locomoción tales como, ruedas, orugas o inclusive patas, además de cámaras y sensores, para identificar su entorno. Algunos robots están provistos de brazos robóticos que les permiten interactuar con el entorno y diversas herramientas en su end-effector para realizar tareas de corte, extracción o manipulación. Desde el software se utilizan técnicas de inteligencia artificial, visión por computador y planificación de rutas (Gildert, 2018).

Se sigue desarrollando investigación en este tipo de sistemas, y algunos de los desafíos que se han identificado tienen que ver con el reconocimiento de frutos y de su madurez; cambios en la intensidad de luz, presencia de polvo, temperaturas extremas y cambios de viento, ya que son sistemas que se encuentran en campo abierto; objetos en movimiento, ya sean animales o personas; manipulación de objetos suaves y delicados, y legislación.

Los vehículos autónomos bien sea UGV o UAV pueden operar en conjunto mediante la robótica cooperativa o sistemas cooperativos. Estos 
se definen como la capacidad que tienen los sistemas inteligentes de coordinar en acción conjunta, para ejecutar procesos a partir de diversos mecanismos subsecuentes (Gildert, 2018). Por ejemplo, al inicio de una misión en una extensión de tierra, un UAV realiza un vuelo en el que inspecciona un área específica, se traza un mapa que luego es procesado para proporcionar una trayectoria al UGV. Mediante un esquema de

\section{Avances en robótica agrícola}

Existen empresas e instituciones universitarias que se dedican a realizar avances en materia de robótica orientada a la agricultura de precisión. navegación en tiempo real, el UAV sobrevuela el área para proporcionar una cobertura global al momento de asistir al UGV en tiempo real para navegar en tierra evitando obstáculos (Cabrera, 2018). Es posible incluir un operador humano para seleccionar puntos de ruta mediante el seguimiento de la cobertura global, aplicando así una arquitectura interactiva en un escenario real (Harik, 2015).

Tabla 1. Descripción de los tipos de robots agrícolas

\begin{tabular}{|c|c|c|}
\hline Función & Descripción & Modelos \\
\hline Recolección y Cosecha & $\begin{array}{l}\text { Robots especializados en la recolección de } \\
\text { frutos o cosecha a gran escala de productos de } \\
\text { granja de consumo masivo. }\end{array}$ & $\begin{array}{l}\text { Agritronics } \\
\text { AGCO Fendt } \\
\text { Robotic Harvesting }\end{array}$ \\
\hline Siembra, Poda, Injertos & $\begin{array}{l}\text { Maquinaria inteligente para realizar una } \\
\text { gestión adecuada del suelo al momento } \\
\text { de sembrar. Son robots con capacidad de } \\
\text { monitorear áreas específicas del cultivo. }\end{array}$ & $\begin{array}{l}\text { Helper Robotech } \\
\text { Conic Systems }\end{array}$ \\
\hline Thinning y Deshierbe. & $\begin{array}{l}\text { Control de población en las plantas sembradas } \\
\text { y manejo de plagas. }\end{array}$ & $\begin{array}{l}\text { Blue River Technologies } \\
\text { Vision Robotics }\end{array}$ \\
\hline $\begin{array}{l}\text { Inspección, Recopila- } \\
\text { ción y Manipulación De } \\
\text { Datos }\end{array}$ & $\begin{array}{l}\text { Mecanismos tipo UAV recorren los cultivos } \\
\text { para recopilar información detallada de las } \\
\text { condiciones del suelo en el espacio tiempo. }\end{array}$ & $\begin{array}{l}\text { PrecisionHawk } \\
\text { senseFly }\end{array}$ \\
\hline $\begin{array}{l}\text { Implementos } \\
\text { Inteligentes }\end{array}$ & $\begin{array}{l}\text { Elementos inteligentes que responden a } \\
\text { comandos desde estaciones de monitoreo. }\end{array}$ & $\begin{array}{l}\text { Autonomous Tractor } \\
\text { Agrobotics AutoProbe }\end{array}$ \\
\hline Automated milking & $\begin{array}{l}\text { Sistemas automatizados para ordeñar vacas, } \\
\text { que optimizan la mano de obra, aumentan la } \\
\text { producción, y mejoran su salud. }\end{array}$ & $\begin{array}{l}\text { Lely } \\
\text { DeLaval VMS }\end{array}$ \\
\hline
\end{tabular}




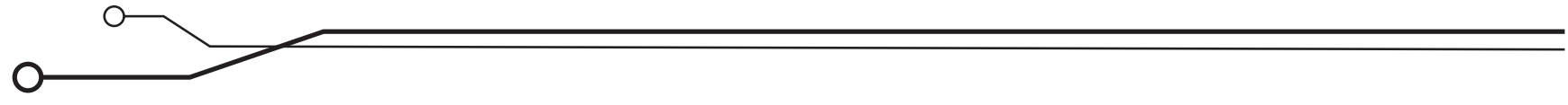

Xaver es un sistema robótico desarrollado por la división Fendt de la empresa AGCO. El sistema consiste en un grupo de robots que operan en enjambre. La administración de datos y satélites en la nube permite planificar, monitorear y documentar con precisión todo lo que respecta a las plantaciones de maíz donde se implemente bien sea con la protección y fertilización de los cultivos, fertilización a cada planta individual, planificación del campo para la siembra de las semillas, la densidad del cultivo, entre otros (Fendt, s. f.).

Figura 3. Módulos que interviene en el funcionamiento de Xaver

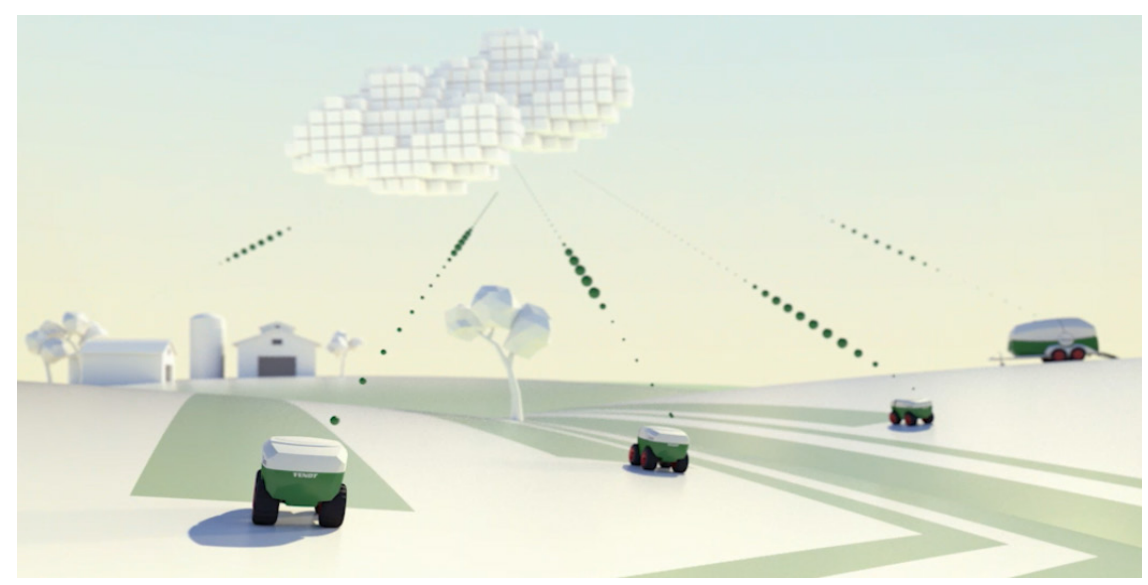

Fuente: tomado de (Fendt, s.f.)

Empresas como Plant Farm, con sede en EE. UU, desarrollan robots recolectores de fresas con capacidad de cosechar 10 hectáreas en tres días. El cosechador, llamado Harvest Croo, utiliza sensores de visión y software para escanear las plantas y localizar las bayas maduras. Su diseño avanzado evita que la fruta sufra cualquier tipo de daño en el proceso de recolección, lo que genera mayores ganancias para los agricultores, al evitar las pérdidas de fruta que normalmente se registran con un recolector humano (Harvest Croo Robotics, s.f.).

Vision Robotics, con sede en San Diego, California, ha desarrollado sistemas robóticos para sembrar la cantidad exacta de lechuga, asegurando una población objetivo para tener el espacio correcto para cada lechuga y prevenir daños por sobrepoblación. Además, la tecnología de visión puede detectar cada planta $\mathrm{y}$, mediante un sistema de suministro, se podrán rociar, nutrir o eliminar lechugas no deseadas si existen problemas de germinación o enfermedad. Todas estas tecnologías, diseñadas por Agmechtronix, también se especializan en la siembra automatizada de cultivos con gestión precisa del espacio del cultivo. Los sistemas automatizados pueden trabajar durante la noche, lo que resuelve el problema de la falta de mano obra en el sector agrícola (Agmechtronix, s.f.).

Otro ejemplo de esta tecnología aplicada al agro es el robot RIPPA, realizado en la Universidad de Sidney, emplea sistemas de tratamiento de imágenes para detectar maleza y remover las plantas no deseadas, además de proporcionar fungicidas en sectores específicos del cultivo para el control de plagas y enfermedades (Dorsey, 2018). 
Figura 4. Robot RIPPA, sistema de reconocimiento de imagen

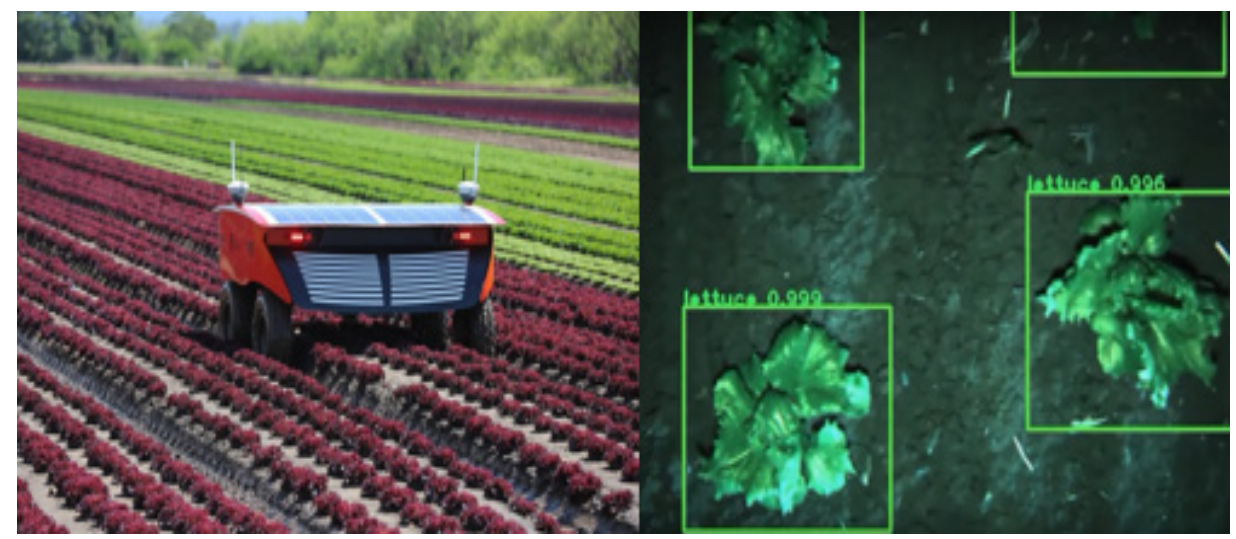

Fuente: construido a partir de Dorsey (2018)

\section{Proyecciones}

Un informe publicado en enero de 2014 por ResearchMoz muestra que el tamaño de mercado para robots agrícolas aumentará de US $\$ 817$ millones, para el 2013, a US \$16.300 millones para el 2020 (ResearchMoz, 2014). Actualmente existen pocos robots en el sector de la agricultura que han alcanzado la comercialización. Sin embargo, AgFunder proyecta que agricultores con más de 200 hectáreas representan un mercado de US $\$ 5500$ millones en el futuro (AgFunder, 2017).

La investigación realizada por IDTechEx afirma que 40000 tractores autónomos no tripulados serán vendidos en 2038 y tractores con algún grado de autonomía tendrán un mercado de US \$27 000 millones. Por el lado de las flotas de robots móviles, la misma investigación afirma que estos sistemas llegarán a ser comercializados en el 2024, con un crecimiento rápido en ventas. Además, incluye que el tamaño de mercado para el 2028 será de US $\$ 900$ millones y en el 2038 de US $\$ 2500$ millones. Los robots recolectores de fruta tendrán su partida en el 2024 y el punto de inflexión se manifestará hasta el 2028; el tamaño de mercado para estos sistemas se proyecta en US $\$ 500$ millones por año. Para los UAV, el tamaño de mercado que proyecta IDTechEx estará sobre los US $\$ 420$ millones en el 2028. Finalmente, IDTechEx afirma que los sistemas robóticos en fincas lecheras ya es un mercado de miles de millones de dólares, siendo instalados miles de estos sistemas alrededor del mundo (Martin, 2018).

Otros reportes, como el realizado por Gran View Research (GVR), muestran que el tamaño del mercado global para robots en el sector de agricultura fue valorado en US \$1050 millones para el 2015. Para ese mismo año, los robots ordeñadores dominaron el mercado en términos de ingresos, siendo el sector lácteo valorado en US \$585,6 millones. Bussines Wire afirma en su reporte que entre el periodo de 2018 a 2023 el mercado global para robots en el sector agropecuario, tendrá una tasa de crecimiento de $18,46 \%$. La valorización del mercado para el 2017 fue estipulada en US \$6374 millones y se proyecta que alcanzará los US \$ 17614 millones para 2023 (Gran View Research, 2017). 


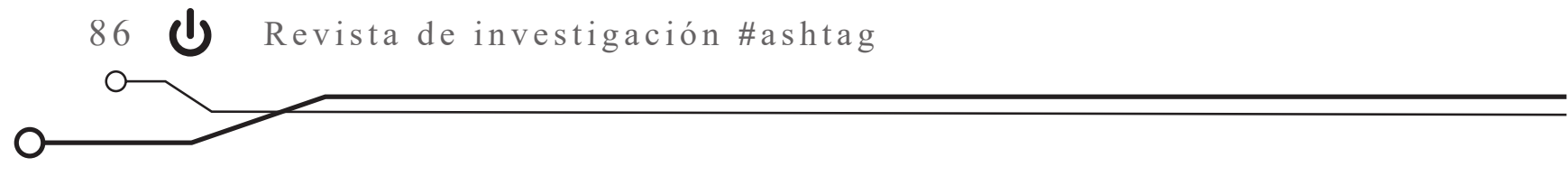

\section{Conclusiones}

El acceso a este tipo de tecnología permitirá que nuevas generaciones permanezcan en el campo debido a mejores ingresos, lo que llevaría a un mayor impacto social en el país. Además, hacer uso de estas herramientas hará que residentes de las zonas rurales sean los operarios de estos sistemas (UAV, UGV, robótica cooperativa), lo que creará nuevos puestos de trabajo acordes a la cuarta revolución y generando inversión social.

La ventaja de Colombia en el sector agropecuario frente a otros países con estaciones climáticas es la de estar cultivando y cosechando durante todo el año; la desventaja es no tener la tecnología apropiada para aumentar su eficiencia para estar entre los mayores productores en el mundo. Desarrollar la agricultura de precisión en Colombia acarreara importantes mejoras en materia de tecnología y le otorgará a la nación reconocimiento ante la comunidad internacional como una potencia agrícola.

Utilizar sistemas autónomos o teleoperados para la fumigación de cultivos, además de hacer esta labor más eficiente, también la hace menos riesgosa para el operario, ya que este no estaría en contacto directo con el herbicida o pesticida mientras es aplicado.

Actualmente se tiene que los países europeos y Estados Unidos son los mayores productores de $\mathrm{UAV}$, con costos para estos sistemas entre los $20 \mathrm{y}$ 30 mil dólares, sumados a los costos por mantenimiento. Por ello, Colombia tiene la oportunidad de diseñar y construir sus propios robots, tanto para el empleo doméstico, como para el exportador.

\section{Referencias}

AgFunder. (2017). AgFunder AgriFood Tech Investing Report - 2017. Recuperado de https:// agfunder.com/research/agfunder-agrifood-tech-investing-report-2017/

Agmechtronix. (s.f.) Automated ag solutions. Recuperado de http:/ / www.agmechtronix.com

Cabrera, F y Barcia, R (2018). Estado del arte de robótica en la agricultura, Revista Observatorio de la Economía Latinoamericana. Recuperado de https://www.eumed.net/rev/oel/2018/05/ robotica-agricultura.html

BLOOMBERG. (29 de julio de 2017). Colombia quiere convertirse en una potencia agroindustrial. Portafolio. Recuperado de https://www.portafolio.co/economia/ colombia-quiere-convertirse-en-una-potencia-agroindustrial-508227

Dorsey, N. (21 de marzo 2018). Top 5 Robotic Systems to Watch in Agriculture- RIPPA. Precision AG. Recuperado de https://www.precisionag.com/in-field-technologies/ top-5-robotic-systems-to-watch-in-agriculture/ 
Fernández, C. (2012). RHEA: un proyecto europeo para el desarrollo de robots aplicados a la protección vegetal, Phytoma, 244, 30-35.

Fendt. (s.f). Proyecto Xaver: Investigación en el campo de la robótica agrícola. Agricultura de precisión - un paso por delante. Recuperado de https://www.fendt.com/es/xaver

Fondo para el Financiamiento del Sector Agropecuario (Finagro). (2018). El momento del Agro. Recuperado de www.finagro.com.co: https://www.finagro.com.co/noticias/ el-momento-del-agro

Gage, D. W. (1995). A Brief History of Unmanned Ground Vehicle (UGV) Development Efforts, Unmanned Systems Magazine, 13(3), 1-7. ISSN: 1792-7625

Gildert, N. (2018). The Need for Combining Implicit and Explicit Communication in Cooperative Robotic Systems, Frontiers in Robotics and AI, 1-6. DOI 10.3389/ frobt.2018.00065

Gran View Research. (2017). U.S. Food Container Market Size, Share \& Trends Analysis Report by Product (Flexible Packaging, Paperboard, Rigid Packaging, Metal, Glass), By Application (Grain Mill, Dairy, Fruits \& Vegetables, Bakery), And Segment Forecasts, 2018 - 2025. Recuperado de https://www.grandviewresearch.com/industry-analysis/ us-food-container-market

Granados, F. L. (2013). Uso de Vehiculos Aereos no tripulados (UAV) para la evaluacion de produccion agraria, Ambienta 105, 40-52.

Harik, C. (2015). UAV-UGv Cooperation for Objects Transportation in an Industrial Area. Recuperado de https:/ / hal.archives-ouvertes.fr/hal-01141993/document

Harvest Croo Robotics. (s.f.). Who we are. Recuperado de https:/ / www.harvestcroo.com/

Keane, J. F. (2013). A Brief History of Early Unmanned Aircraft. Johns Hopkins Apl Technical Digest.

Martin, C. (Junio 2018). Global Agricultural Robots and Drones Forecast 2018 - 2038: Technologies, Markets and Players. Recuperado de https:/ / www.prnewswire.com/news-releases/ global-agricultural-robots-and-drones-forecasts-2018-2038-technologies-markets-and-players-300674238.html

Mejía, R. (2016). ¿Se está promoviendo la inversión agrícola en Colombia? Revista Nacional de Agricultura. Recuperado de https://sac.org.co/ se-esta-promoviendo-la-inversion-agricola-en-colombia/ 


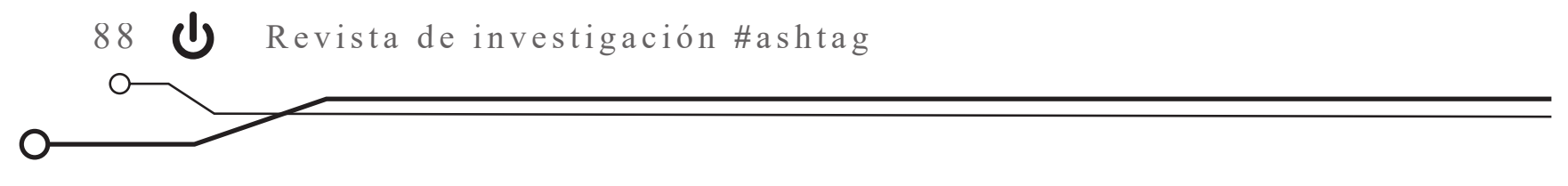

El Tiempo. (23 de mayo de 2016). El $65.8 \%$ de la tierra apta para sembrar en Colombia no se aprovecha. El Tiempo. Recuperado de https://www.eltiempo.com/archivo/documento/ CMS-16601436

Oficina de información científica y tecnológica para el congreso de la unión. (2018). Agricultura de Precisión. (15). Recuperado de https://www.foroconsultivo.org.mx/INCyTU/documentos/Completa/INCYTU_18-015.pdf

Organización de las Naciones Unidas para la Alimentación y la Agricultura (FAO). (2017). Concentración y extranjerización de tierras productivas en Colombia. Bogotá. ISBN: 978-92-5-309941-2

ResearchMoz (2014). Global Agricultural Robots Market - Shares, Strategies, and Forecasts, Worldwide, 2014 to 2020 Report Available Online by ResearchMoz.us. Recuperado de https:/ / www.prnewswire.com/news-releases/global-agricultural-robots-market--shares-strategies-and-forecasts-worldwide-2014-to-2020-report-available-online-by-researchmozus-242551581.html

Unimedios. (30 de diciembre de 2015). Envejecimiento de habitantes rurales, otro freno al desarrollo del campo. Recuperado de https://agenciadenoticias.unal.edu.co/detalle/article/ envejecimiento-de-habitantes-rurales-otro-freno-al-desarrollo-del-campo.html 